\title{
PRODUCCION CIENTIFICA ESPAÑOLA SOBRE SALUD LABORAL INDIZADA EN EL ÍNDICE MÉDICO ESPAÑOL (IME)
}

\author{
JULIO ALONSO ARÉVALO *, SONIA MARTÍN CASTILLA**, \\ HELENA MARTÍN RODERO***
}

(*) Biblioteca de la Facultad de Traducción y Documentación. Universidad de Salamanca.

(**) Biblioteca de la Escuela Universitaria de Enfermería y Fisioterapia. Universidad de Salamanca.

(***) Biblioteca de la Facultad de Medicina. Universidad de Salamanca.

\section{RESUMEN}

Objetivo: evaluar la producción científica española en salud laboral entre los años 1971 y 2007 a partir de los datos recogidos en la base de datos IME. Establecer las características relativas a la autoría, filiación y nivel de cooperación de los investigadores y determinar cuáles son las materias emergentes y cuáles son las áreas de interés principales.

Método: a través de la metodología de búsqueda derivada se ha realizado un barrido, lo más exhaustivo y pertinente posible, en la base de datos IME de los registros sobre salud laboral. Los datos obtenidos han sido exportados y analizados a través del gestor de referencias ProCite. Mediante el tabulado de los datos se realiza un estudio de la autoría y filiación, y se efectúa un análisis de series temporales por descriptores y categorías.

Resultados: 1530 referencias de documentos publicados procedentes de 180 revistas, congresos y partes de libros. El promedio de autores es de 2,6 autores por artículo. Un tercio de los autores sólo ha publicado un artículo sobre el tema. Las materias más estudiadas pertenecen al ámbito de la psicología del trabajo.

Conclusiones: se constata un alto nivel de cooperación entre autores, así como una baja especialización, tanto en la autoría, como en las revistas en las que se publican los trabajos. Se observa una curva ascendente en los temas relativos al campo de la psicología del trabajo, con un notable incremento en los últimos cuatro años.

\section{PALABRAS CLAVES}

Salud laboral/ Producción científica/ España/ IME/Gestores de referencias bibliográficas/ Psicología del trabajo.

\section{ABSTRACT}

The aims of this paper are: 1) to evaluate Spanish scientific publications on Occupational Health between 1971 and 2007, based on data collected from the IME database; 2) to present the features related to authorship, and the connections and potential cooperation among researchers; and 3) to determine emerging topics and main areas of interest.

Through a derived search methodology, we have carried out an exhaustive scanning of IME records on Occupational Health, selected according to relevance. The results of this search were exported and analysed with the ProCite reference manager. This procedure allowed us to perform a survey of authorship and connections, and an analysis of temporal sets by descriptors and categories.

The results are 1,530 references of public documents from 180 scientific journals, conferences and book chapters. The average figure of authors per article is 2.6. One third of the authors published only one article on the topic. The subject most frequently studied belongs to the field of Organizational Psychology.

The conclusions we have arrived at are: 1) there is a high level of cooperation among authors, a low level of specialisation, as it refers both to authorship and to journals where scientific papers are published; 2) a rising trend in the field of Organizational Psychology, with a clear increase in this area during the last four years

\section{KEY WORDS}

Key Words: Occupational Health/Scientific publications/IME/Citation reference managers/ Organizational Psychology. 


\section{SALUD LABORAL EN ESPAÑA}

En el momento actual estamos asistiendo a un proceso de especial sensibilidad social y sanitaria, y a un compromiso político en el campo de la seguridad y salud en el trabajo.

A partir de 1995, con la promulgación de la Ley 31/1995 de 8 de noviembre, de Prevención de Riesgos Laborales (1) se empieza a observar una actitud de cambio en España. La prevención de los riesgos laborales pasó de ser una disciplina desconocida y reservada a especialistas a consolidarse como un aspecto fundamental de las relaciones laborales. Durante estos años España se ha ido acercando a las políticas de seguridad y salud en el trabajo de la Unión Europea y a las políticas desarrolladas por sus Estados miembros.

En el 2004 el gobierno se comprometió con los agentes sociales, fuerzas políticas y comunidades autónomas a impulsar acciones tendentes a reducir la siniestralidad laboral y mejorar de forma continua los niveles de seguridad y salud en el trabajo. Esto se plasmó en el documento "Estrategia española en materia de Seguridad y Salud en el Trabajo 2007-2012” (2).

En el año 2005 nace el Observatorio de Salud Laboral como respuesta a la necesidad de disponer de información de calidad que ayude a la toma de decisiones estratégicas en los distintos ámbitos de la seguridad y la salud en el trabajo, tanto en el campo científico como en el profesional, social y político.

En el momento actual, más que nunca, es preciso desarrollar y fortalecer los sistemas de información sanitaria en salud laboral para conocer las causas de las enfermedades profesionales, el componente de exposición ocupacional de los sucesos centinela, mejorar las fuentes de datos sobre exposición y daños para la salud, y realizar actividades de promoción de la salud.

En este sentido, los profesionales de la salud laboral en España necesitan información para poder identificar los factores de riesgo a los que la población laboral está expuesta, establecer sistemas de vigilancia epidemiológica, y planificar y evaluar la actividad preventiva y asistencial.

Por este motivo, es necesario realizar un estudio sobre el estado actual de la producción científica española en este ámbito.

Existe un estudio previo desarrollado por $\mathrm{N}$. Alba, F. Abad y C. García (3), donde se realiza un análisis de la producción científica en Salud Laboral a partir de la base de datos Índice Médico Español, que aunque tiene una antigüedad de más de 12 años, nos ha sido de gran ayuda por su apartado metodológico.

\section{METODOLOGÍA DE TRABAJO}

Todo trabajo analítico-sintético de carácter bibliométrico como es el que aquí presentamos debe apoyarse en un depurado planteamiento metodológico, ya que la calidad última de los resultados obtenidos está condicionada por la aplicación de un método bien definido.

La metodología se puede establecer en tres fases:

1. Recogida de de datos

2. Tabulación y análisis de datos

3. Extracción de conclusiones

\section{Recogida de datos}

La fuente objeto de nuestro análisis ha sido la base de datos IME (Índice Médico Español) producida y elaborada por el Consejo Superior de Investigaciones Científicas (CSIC). El conjunto de bases de datos del CSIC forma la recolecta más exhaustiva de la investigación española en todos los campos del conocimiento. Se trata de un recurso de primera elección para estudios de este tipo, ya que la presencia de la producción científica de nuestro país en las bases de datos de carácter internacional tiene un carácter poco representativo. Además, las bases de datos nacionales recogen referencias de artículos que, aunque no tengan un impacto internacional, sí lo tienen en el ámbito nacional o regional. Por otro lado, aunque existen otras bases de datos en español de ciencias de la salud, éstas no permiten la exportación de su contenido con la misma facilidad que el IME.

Las bases de datos CSIC (4) contienen más de 1.250.000 referencias bibliográficas de artículos publicados en más de 2.630 revistas especializadas editadas en España en las principales Áreas Científicas y Técnicas. El paquete incorpora las bases de datos siguientes:

ICYT: base de datos bibliográfica que recoge y analiza más de 600 publicaciones periódicas españolas, dentro de los campos de la Ciencia y la Tecnología desde 1979.

IME: base de datos bibliográfica del área biomédica que recoge y analiza más de 330 revistas médi- 
cas españolas desde 1971 sobre Administración Sanitaria, Farmacia, Clínica, Medicina Experimental, Microbiología, Psiquiatría y Salud Pública.

ISOC: base de datos bibliográfica que recoge y analiza más de 1.600 revistas españolas relativas a las Ciencias Sociales y Humana desde 1971.

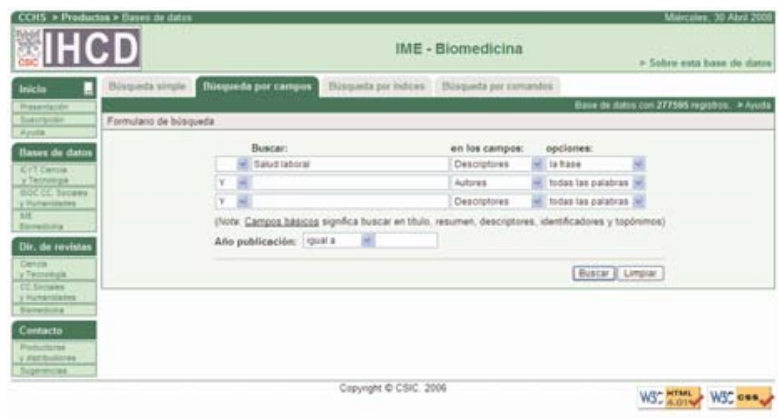

Figura 1. Base de datos CSIC-IME.

El objetivo de esta fase es recoger toda la información de la manera más exhaustiva y pertinente posible, con el fin de que el estudio sea totalmente representativo de la producción científica en medicina del trabajo y salud laboral. Para ello hemos utili- zado la posibilidad de búsqueda avanzada que nos ofrece IME. La metodología utilizada ha sido la técnica documental denominada búsqueda derivada, ya que aunque la base de datos sí contiene descriptores, no permite la utilización de un tesauro que agrupe bajo un descriptor único y genérico todas las categorías posibles. La búsqueda derivada consiste en la recuperación, mediante lenguaje natural, de los registros más relevantes sobre un tema determinado, para posteriormente, utilizar los descriptores asignados a esos registros. De esta manera hemos realizado alrededor de una treintena de búsquedas que han supuesto un barrido completo del contenido de la base de datos.

Se han recuperado 1530 referencias de artículos de revista, actas de congresos y partes de libros del campo de salud laboral producidos en España entre los años 1971 y 2007, años de cobertura proporcionados por el Índice.

Para la compilación de los datos hemos utilizado el gestor de referencias bibliográficas ProCite, una herramienta flexible y completa que ofrece la posibilidad de importar las referencias en formato RIS. Este formato es una de las opciones de exportación que ofrecen las grandes bases de datos en el ámbito internacional. Una cuestión muy importante para la importación de las diferentes búsquedas es la posibilidad de detectar duplicados, pues muchos de los descriptores compartían los mismos artículos; esta opción asegura la limpieza de la base de datos.

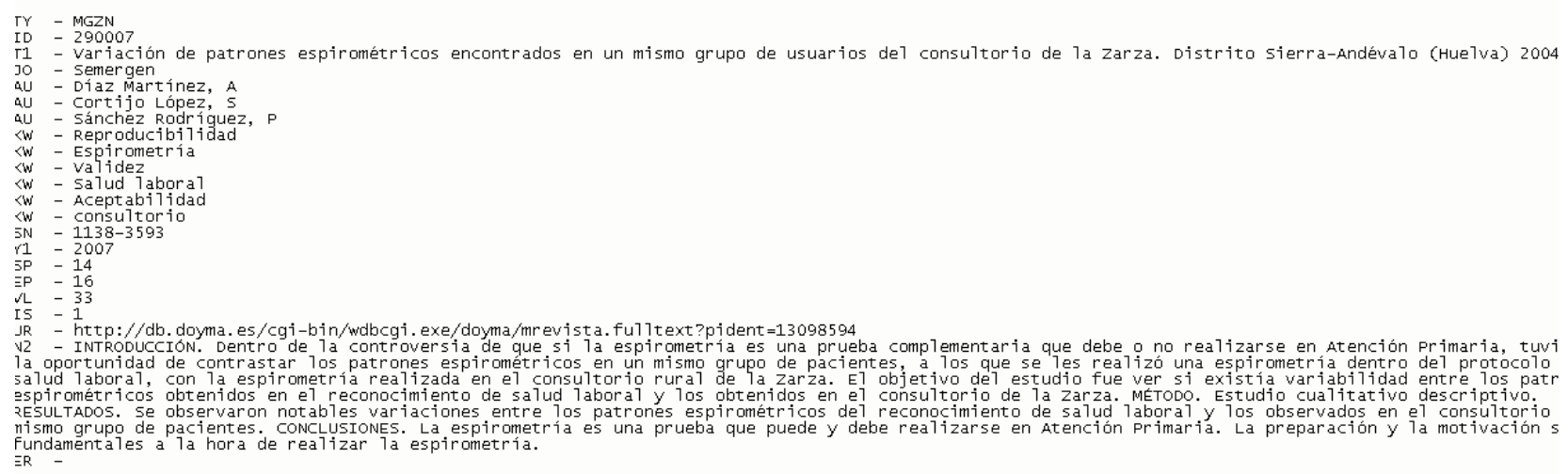

Figura 2. Formato RIS.

Las referencias obtenidas tienen una estructura de registro con los siguientes campos: autor, título, fuente, año de publicación, y en su caso, volumen, número y páginas, filiación de los autores, localización URL, ISBN o ISNN de la publicación, resumen y palabras clave. (Ver figura 3 ). 


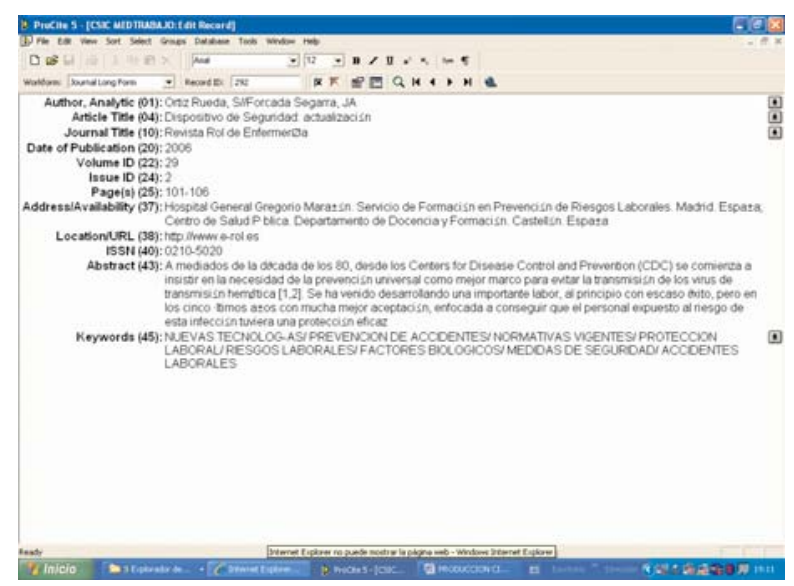

Figura 3. Base de datos ProCite.

\section{Tabulación y análisis de datos}

La base de datos generada por ProCite proporciona automáticamente índices de autores, títulos, fuentes y descriptores, indicando la frecuencia de aparición. Esto ofrece la posibilidad de realizar tablas y gráficos. (Ver figura 4)

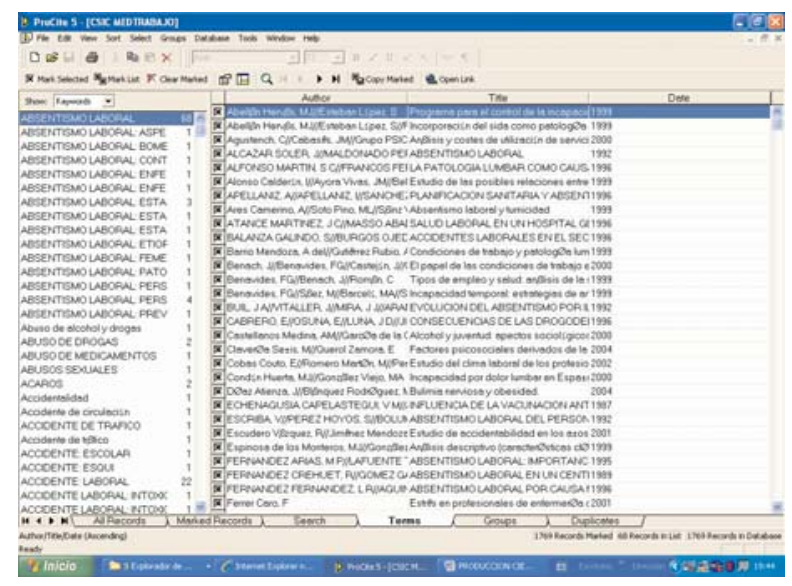

Figura 4. Índices ProCite.

Los datos relativos a autores los hemos obtenido por medio de la opción "Print subject bibliography". De esta manera, seleccionando el campo de autores, se genera una lista del número total de todos ellos, con la frecuencia de aparición de los mismos, generando así un listado de autores con el número de artículos publicados por cada uno de ellos.

Otra cuestión interesante es conocer cuál es el peso de la cooperación entre autores: distinguir qué porcentaje publican individualmente frente a aquellos que lo hacen de una manera colaborativa; entre estos últimos se distingue autoría compartida (dos autores) y autoría múltiple (más de dos autores).

Un aspecto de especial interés es identificar cuáles son los autores más prolíficos en esta área de conocimiento y su filiación profesional, para conocer las instituciones que mayor empeño están desarrollando en la investigación en salud laboral.

El otro apartado de análisis se refiere a cuáles son las áreas de interés de los autores que investigan y publican en Salud Laboral, conocer cuáles son las materias emergentes y cómo ha evolucionado la disciplina a lo largo de los años. Para este trabajo hemos generado tablas sobre las materias proporcionadas por IME y su frecuencia de aparición. Hay que poner de manifiesto que la consistencia en la asignación de descriptores no tiene una buena coherencia interna; por ello ofrece una visión más rigurosa el establecer grupos de materias o categorías en función de las grandes áreas que configuran la investigación en salud laboral. Para ello hemos utilizado la opción del gestor denominada "Groups", que nos permite trabajar con grupos de materias en la misma base de datos, pero haciendo análisis específicos por cada uno de ellos.

\section{Extracción de conclusiones}

Tras recopilar los registros y exportarlos al gestor de referencias es fundamental realizar un análisis crítico y objetivo de los resultados. Para ello se han recogido los datos obtenidos, se han cruzado datos relativos autores y materias, y se han desarrollado series temporales para determinar cómo se han producido los cambios y evolución en esta área. A partir de los datos se han generado tablas y gráficos para facilitar su análisis y establecer conclusiones.

\section{ANÁLISIS DE DATOS}

La población estudiada está compuesta por 1530 artículos relativos a salud laboral recogidos por medio de una treintena de búsquedas de aquellas materias relevantes en esta área.

\subsection{Autoría}

El número total de autores recogidos en nuestra muestra son 4.074, lo que supone una media de 2,6 autores por artículo. Se trata de un indicador que pone de manifiesto un alto nivel de cooperación. La importancia de la autoría individual es baja, ya que el número de artículos firmados por un solo autor 
es de 434 referencias. Es decir, un $28 \%$ de autoría individual frente al $72 \%$ de artículos en los que colaboran dos o más autores. El peso de la autoría compartida frente a la múltiple es también bajo. Este es un dato muy positivo: la cooperación en la investigación es esencial, pues implica la existencia de una aportación más compleja y la existencia de puntos de vista más amplios que han sido previamente discutidos y consensuados entre los autores.

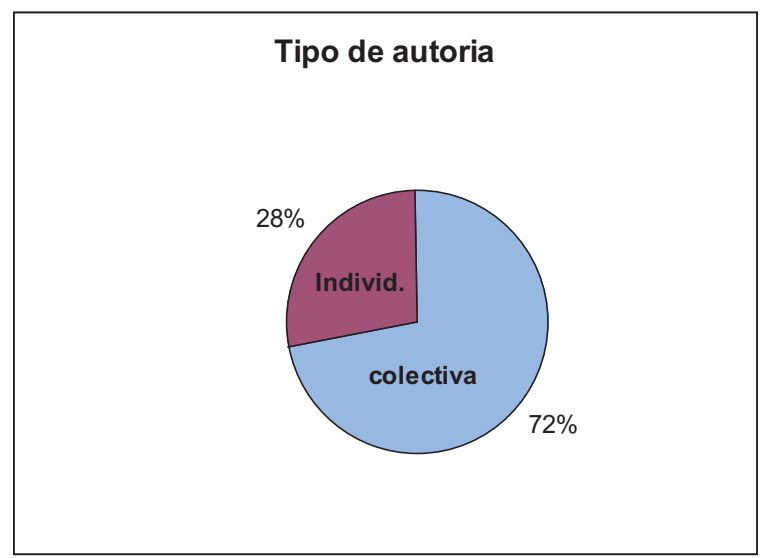

Figura 5. Autoría.
Otra cuestión de interés es conocer qué autores han sido más productivos y cuál es su filiación, es decir, de qué entidades proceden, así como conocer los porcentajes de autores que publican más artículos. Es muy significativo que el $71 \%$ de los autores solamente ha publicado un artículo sobre el tema; esto indica que el nivel de especialización no es muy alto. Solamente un $19 \%$ (283 autores) han publicado 2 artículos sobre la cuestión; un $6 \%$ ha publicado más de 3 artículos y solamente un $2 \%$ ha publicado más de tres.

\begin{tabular}{ccc}
\hline Numero de artículos & Numero de autores & $\mathbf{\%}$ \\
\hline 17 & 2 & $0 \%$ \\
\hline 12 & 1 & $0 \%$ \\
\hline 11 & 1 & $0 \%$ \\
\hline 10 & 3 & $0 \%$ \\
\hline 9 & 2 & $0 \%$ \\
\hline 8 & 3 & $0 \%$ \\
\hline 7 & 5 & $0 \%$ \\
\hline 6 & 7 & $0 \%$ \\
\hline 5 & 15 & $1 \%$ \\
\hline 4 & 33 & $2 \%$ \\
\hline 3 & 86 & $6 \%$ \\
\hline 2 & 283 & $19 \%$ \\
\hline 1 & 1089 & $72 \%$ \\
\hline
\end{tabular}

Figura 6. Número de artículos por autor

\section{AUTOR}

RODRÍGUEZ ORTIZ DE SALAZAR (17 docs.)

BENAVIDES, FG (17 docs.)

GÓMEZ-CONESA, A (12 docs.)

ARTAZCOZ, L (11 docs.)

CONDE-SALAZAR, L (10 docs.)

IDOATE GARCÍA, VM (10 docs.)

ARTIEDA, L (10 docs.)

BENACH, J (9 docs.)

ARES CAMERINO, A (9 docs.)

ARRIBAS LLORENTE, J. L.

\section{ENTIDAD}

Escuela Nacional de Medicina del Trabajo. Instituto de Salud Carlos III. Madrid

Universitat Pompeu Fabra. Barcelona

Facultad de Medicina. Departamento de Fisioterapia. Murcia

Institut Municipal de la Salut. Barcelona

Inst.Nac.Hig.Segur.Trabajo, Serv.Dermatologia, Madrid

Instituto Navarr o de Salud Laboral. Pamplona.

Navarra

Instituto Navarro de Salud Laboral. Pamplona

Unidad de Investigación en Salud Laboral.

Universidad Pompeu Fabra. Barcelona. España

Diputación Provincial. Unidad de Salud Laboral. Cádiz. España

Hosp.Miguel Servet, Serv.Med.Prevent.yY Soc., Zaragoza 


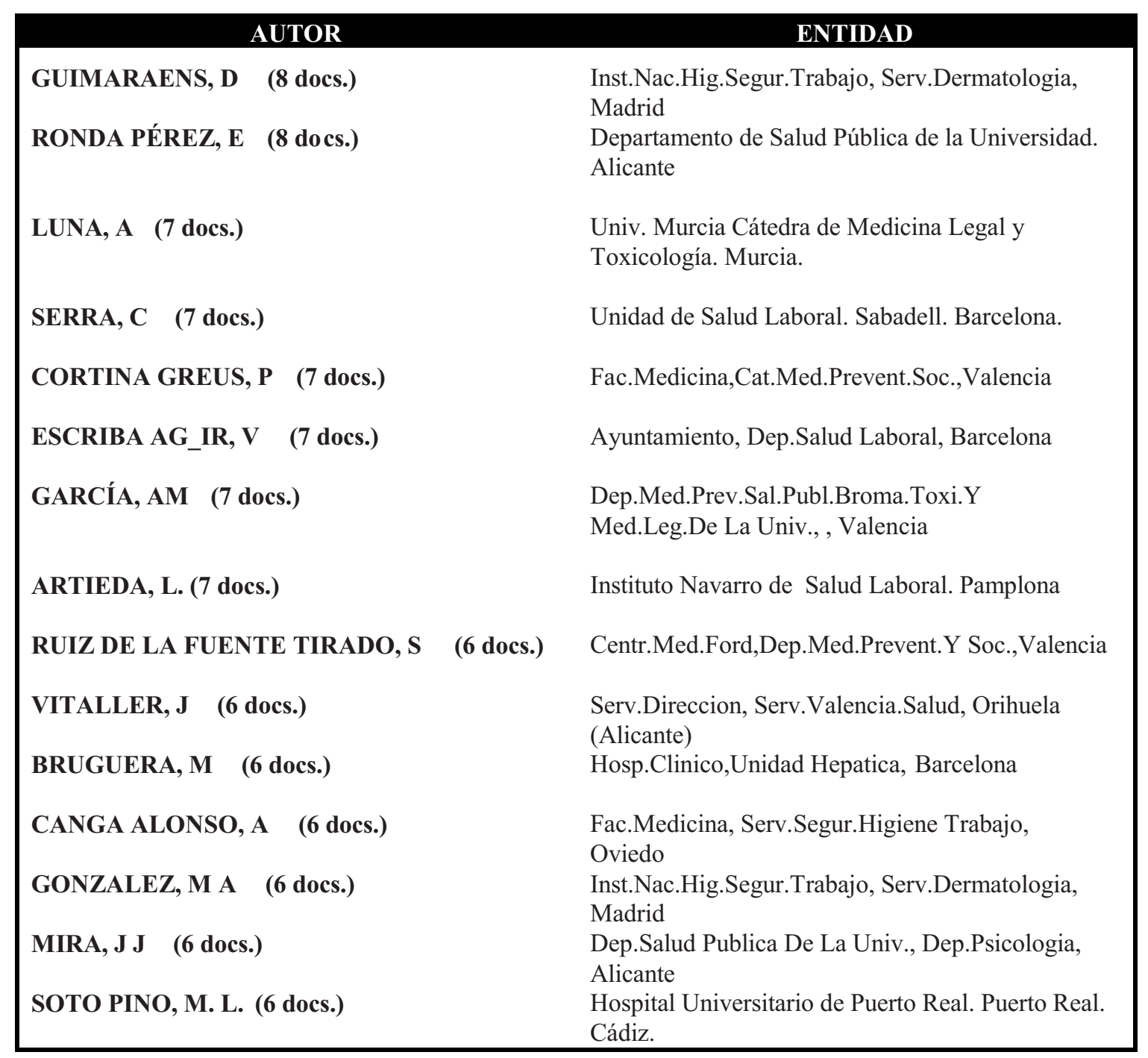

Figura 7. Lista de autores por $n^{\circ}$ de artículos publicados

Sería deseable que la base de datos IME normalizara los nombres de las instituciones; aparecen un total de 998 entidades, pero esto no tiene una base real, ya que las diferentes entidades aparecen de diversas formas. Por ello hemos optado por establecer un ranking de tipos de entidades, tales como universidades, departamentos de salud de diversas instituciones, institutos de investigación y centros de salud, etc. Hay que decir que no todas las referencias incluyen la adscripción de los investigadores, sólamente 400 de los 1530 documentos utilizados para este estudio.

El tipo de organismo que más publica sobre salud laboral pertence al ámbito hospitalario, con un 39\%, seguido por las universidades con un $22 \%$; a

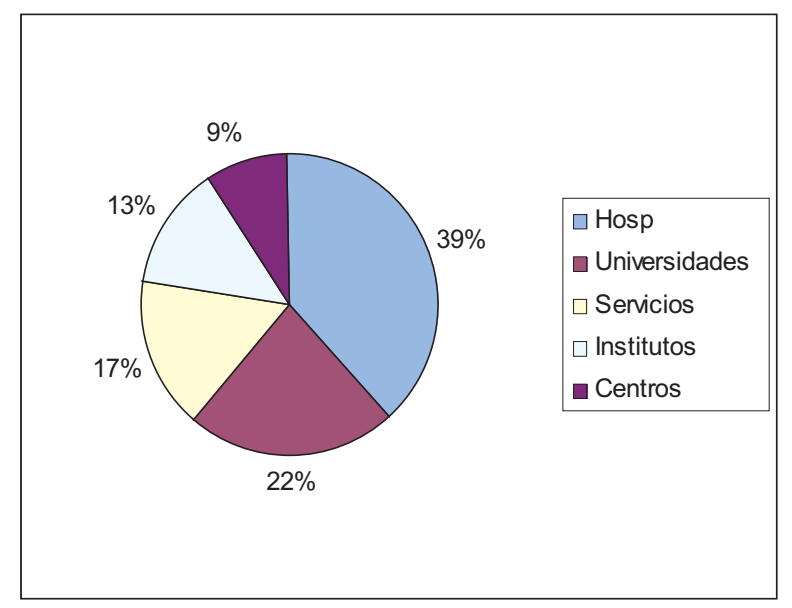

Figura 8. Institución de procedencia de los autores 
continuación están los servicios, institutos y centros de salud, en este orden. Por los datos podemos deducir la importancia del sector profesional sobre el docente.

\subsection{Series temporales}

Otro aspecto relevante para el estudio es considerar cómo ha sido la evolución de la producción científica de salud a lo largo del tiempo, es decir, si el número de estudios sobre salud laboral se ha ido incrementado entre 1971 y 2007 . Tal y como se constata en la figura 9, el principal incremento y toma de conciencia sobre la información en salud laboral en nuestro país se produce a partir de la segunda mitad de los años 90, que es cuando el número de artículos por año empieza a superar el centenar. Este hecho está debido seguramente a que en 1995 se promulgó la Ley de Prevención de Riesgos Laborales.

Este promedio se mantiene hasta la actualidad, salvo algún año anómalo como es el año 1999 en el que el número total de artículos supera con creces la media, con 232 documentos sobre la cuestión. No es significativa la aparente caída que aparece en el año 2007, ya que es el último año en curso, y aún no se ha recopilado en la base de datos IME todo lo publicado ese año.

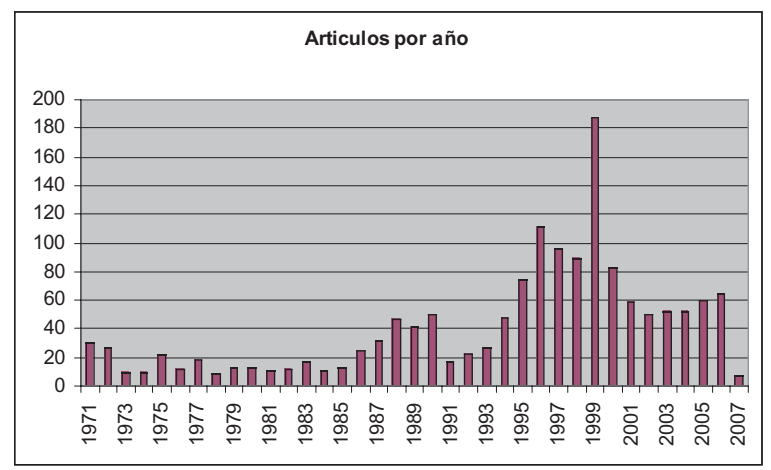

Figura 9. Artículos por año.

Probablemente es más significativo observar esta evolución por décadas. Vemos en el gráfico adjunto cómo ha habido un incremento continuo de la producción científica en salud laboral; llama la atención el hecho de que la década del 2000, que aún no ha finalizado, sigue una pauta ascendente, según la cual es posible que la producción pueda casi duplicarse.

\begin{tabular}{|c|c|}
\hline Década & Artículos \\
\hline $1970-1979$ & 153 \\
\hline $1980-1989$ & 222 \\
\hline $1990-1999$ & 726 \\
\hline $2000-2006$ & 427 \\
\hline
\end{tabular}

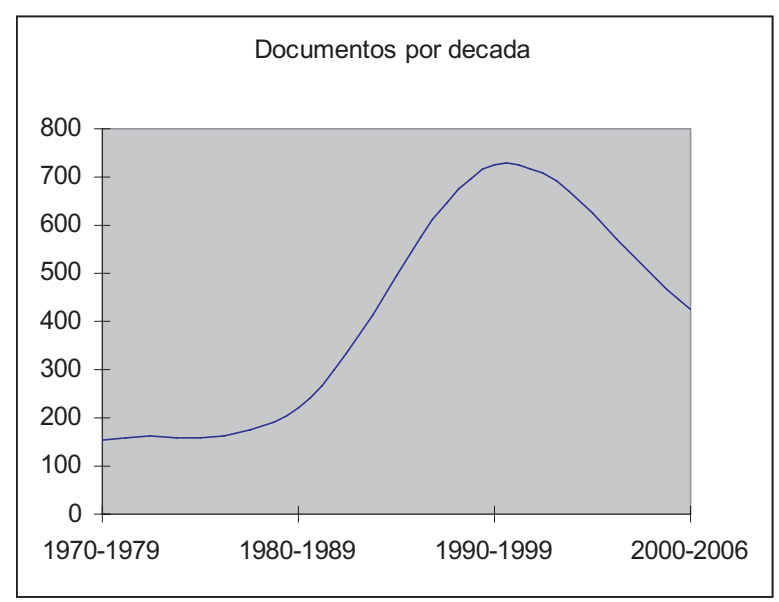

Figura 10. $N^{o}$ de documentos por década.

\subsection{Publicaciones fuente}

Las revistas españolas que incluyen un mayor número de artículos totales del tema de nuestro estudio son las siguientes:

\begin{tabular}{|c|c|}
\hline TíTULO & $\begin{array}{c}\mathbf{N}^{\mathbf{0}} \text { DE } \\
\text { ARTÍCULOS }\end{array}$ \\
\hline $\begin{array}{c}\text { Medicina y seguridad del } \\
\text { trabajo (5) }\end{array}$ & 276 \\
\hline Medicina clínica (6) & 77 \\
\hline Atención primaria (7) & 76 \\
\hline Archivos de prevención (8) & 73 \\
\hline $\begin{array}{c}\text { Revista española de salud } \\
\text { pública (9) }\end{array}$ & 63 \\
\hline Gaceta sanitaria (10) & 49 \\
\hline
\end{tabular}

Figura 11. Revistas fuente. 
Este dato ha de ser tenido en cuenta por el personal interesado en el tema, ya que el especialista en salud laboral no ha de limitarse sólo a la revisión de las publicaciones especializadas en su campo, sino que tendrá que tener en cuenta publicaciones de carácter general como Medicina Clínica y Atención Primaria. Hay que destacar que todas estas revistas ya cuentan con versión electrónica que ofrece acceso gratuito al texto completo, o al menos a una parte de la colección.

\subsection{Materias emergentes}

Es importante conocer cuáles son las materias que despiertan mayor interés entre los investigadores, saber cómo han evolucionado en el tiempo y a qué razones obedece el cambio de centro de gravedad en las áreas estudiadas. Para ello hemos utilizado la posibilidad que nos ofrece el gestor de referencias ProCite, que crea un índice de descriptores con su frecuencia de aparición en la base de datos.
Este análisis entraña una cierta dificultad, pues IME no utiliza un tesauro que nos permita categorizar cuáles son las áreas de interés de una manera objetiva; además el alto número de descriptores utilizados para la indización, 2689 para el total de los artículos, indica una falta de homogeneización en la descripción.

Debido a la escasa coherencia de la indización por descriptores que ofrece la base de datos, hemos establecido cuatro categorías que nos permitirán realizar un estudio de la cobertura temática de los registros seleccionados. Hay que tener en cuenta que dentro de una categoría un registro puede pertenecer a más de un grupo (p. ej. un estudio sobre cáncer de pulmón puede estar encuadrado dentro de la categoría 205 y la categoría 22). Los grupos se han establecido tomando como base el tesauro ILOCIS(11), la Clasificación Decimal Universal y el CIUO-88(12).

Categoría 1. Clasificación según el tema general del artículo:

\begin{tabular}{|l|c|c|}
\hline \multicolumn{1}{|c|}{ GRUPO } & N $^{\mathbf{0}}$ DE ARTÍCULOS & $\mathbf{\%}$ \\
\hline 11. Estudios de riesgos & 282 & 18.43 \\
\hline 12. Estudios sobre prevención & 211 & 13,79 \\
\hline 13. Estudios de valoración & 135 & 8.82 \\
\hline 14. Legislación y administración & 119 & 7,77 \\
\hline 15. Aspectos socioeconómicos & 95 & 6.20 \\
\hline 16. Epidemiología & 190 & 12.41 \\
\hline 17. Psicología del trabajo & 250 & 16,33 \\
\hline 18. Estudios específicos & 249 & 16,27 \\
\hline 19. Otros & 75 & 4,90 \\
\hline
\end{tabular}

El grupo 11 incluye estudios sobre factores de riesgo, exposición ocupacional, condiciones de trabajo, mapas de riesgos, etc. El 12, estudios sobre prevención, ergonomía, vacunación e inmunoprofilaxis, protección y autoprotección, vigilancia, promoción de la salud y educación sanitaria en el ámbito laboral, dispositivos de seguridad, diagnóstico precoz... El 13 abarca lo relativo a estudios de valoración, medición, pruebas clínicas, baremos, cuestionarios, escalas, encuestas, peritaje y afines. El 14, aspectos de derecho sanitario, administración, normativas, sanciones, prestaciones, baja y absentismo laboral, etc. Los artículos relativos a estudios de costes, gastos y al impacto social de los accidentes y enfermedades laborales están agrupados en el grupo 15. El 16 comprende los documentos sobre epidemiología de enfermedades y accidentes laborales, prevalencia, mortalidad, morbilidad, incidencia o incapacidad laboral. El 17, la psicología del trabajo en sentido amplio (condiciones de trabajo, adaptación al entorno, satisfacción laboral, estrés profesional, etc.) El grupo 18 está formado por estudios específicos (médicos) de una enfermedad determinada. Por último se ha creado un grupo 19 para todos aquellos artículos que no han podido ser incluidos en ninguna de las categorías anteriores.

Para conocer las áreas emergentes hemos considerado el análisis detallado de los años 2004 a 2007. Es muy significativo que en el grupo 17 (Psicología del trabajo) el 20,04\% de los artículos ha sido publicado en este último periodo, es decir, una cuarta parte del total. El resto de grupos tienen una distribución similar entre ellos, oscilando entre el 12\% y el $15 \%$, excepto los estudios sobre epidemiología, que caen al 7,36\%. 


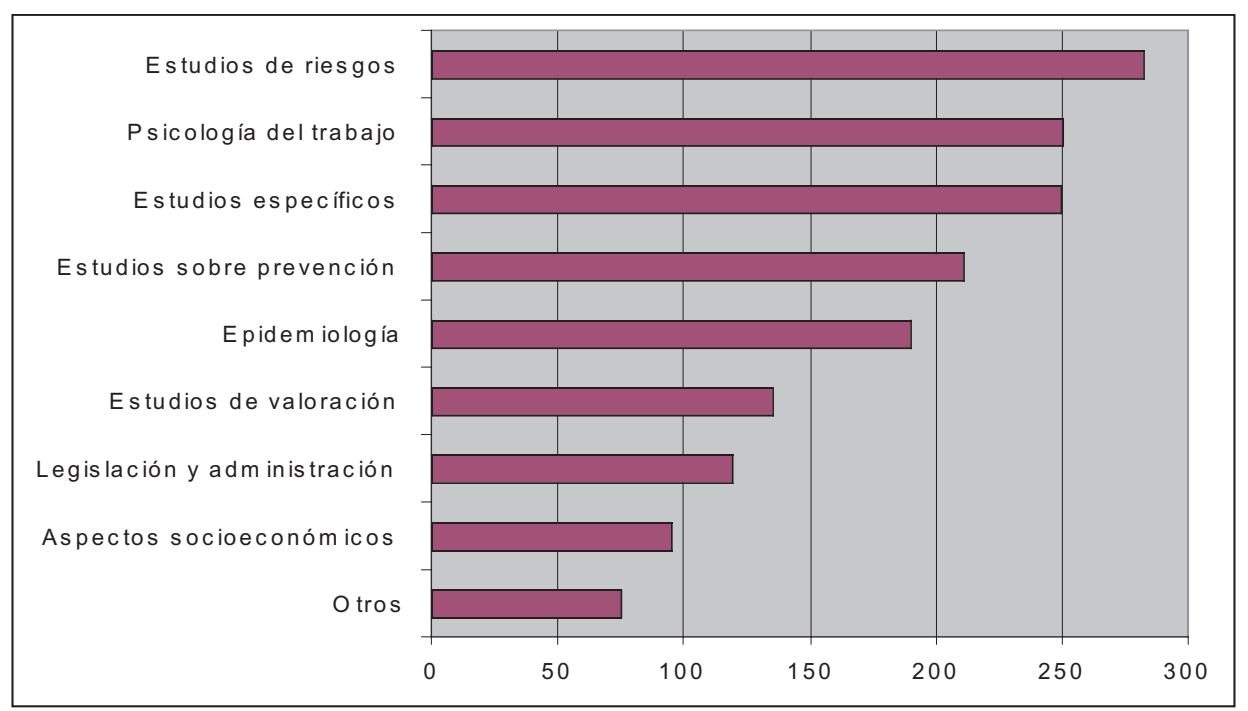

Figura 12. Categoría 1

Categoría 2. Tipos de enfermedades y órganos o aparatos afectados

\begin{tabular}{|l|c|}
\hline \multicolumn{1}{|c|}{ GRUPO } & $\mathrm{N}^{\circ}$ DE REGISTROS \\
\hline 201. Oftalmología & 12 \\
\hline 202. Ginecología. Obstetricia. Maternal & 32 \\
\hline 203. Trastornos psicológicos y psiquiátricos & 151 \\
\hline 204. Alergia & 64 \\
\hline 205. Cáncer & 45 \\
\hline 206. Intoxicaciones & 61 \\
\hline 21. Enfermedades cardiovasculares & 53 \\
\hline 22. Enfermedades respiratorias & 105 \\
\hline 221. Enfermedades otorrinolaringológicas & 30 \\
\hline 23. Enfermed ades del aparato digestivo & 21 \\
\hline 24. Trastornos del metabolismo & 9 \\
\hline 25. Enfermedades de la piel: & 54 \\
\hline 26. Enfermedades genito -urinarias y nefrológicas & 11 \\
\hline 27. Afecciones del aparato locomotor. Traumatología & 132 \\
\hline 28. Trastornos neurológicos & 29 \\
\hline 29. Infecciones & 111 \\
\hline
\end{tabular}

Dentro de la segunda categoría el grupo más numeroso es el 203 (Trastornos psicológicos y psiquiátricos), con 151 registros, seguido del grupo 22 (Aparato respiratorio) con 105 registros. Pero mientras en el grupo 203 , el $21,12 \%$ de los estudios ha sido publicado entre los años 2004 y 2007, lo cual nos permite clasificarlo como una materia con interés relevante en la actualidad, en el grupo 22 tan sólo han sido publicados en este mismo periodo de tiempo el $4,76 \%$ de los artículos. Esto puede ser debido a que la mayor parte de los estudios del aparato respiratorio son los relativos a las enfermedades profesionales de la minería, sector económico que ha disminuido su actividad en España en los últimos años de manera radical.

El trastorno más estudiado en el ámbito de la psicología es el de estrés laboral o burnout, con un total de 137 registros, el 25\% de los cuales se ha publicado en los últimos cuatro años. 


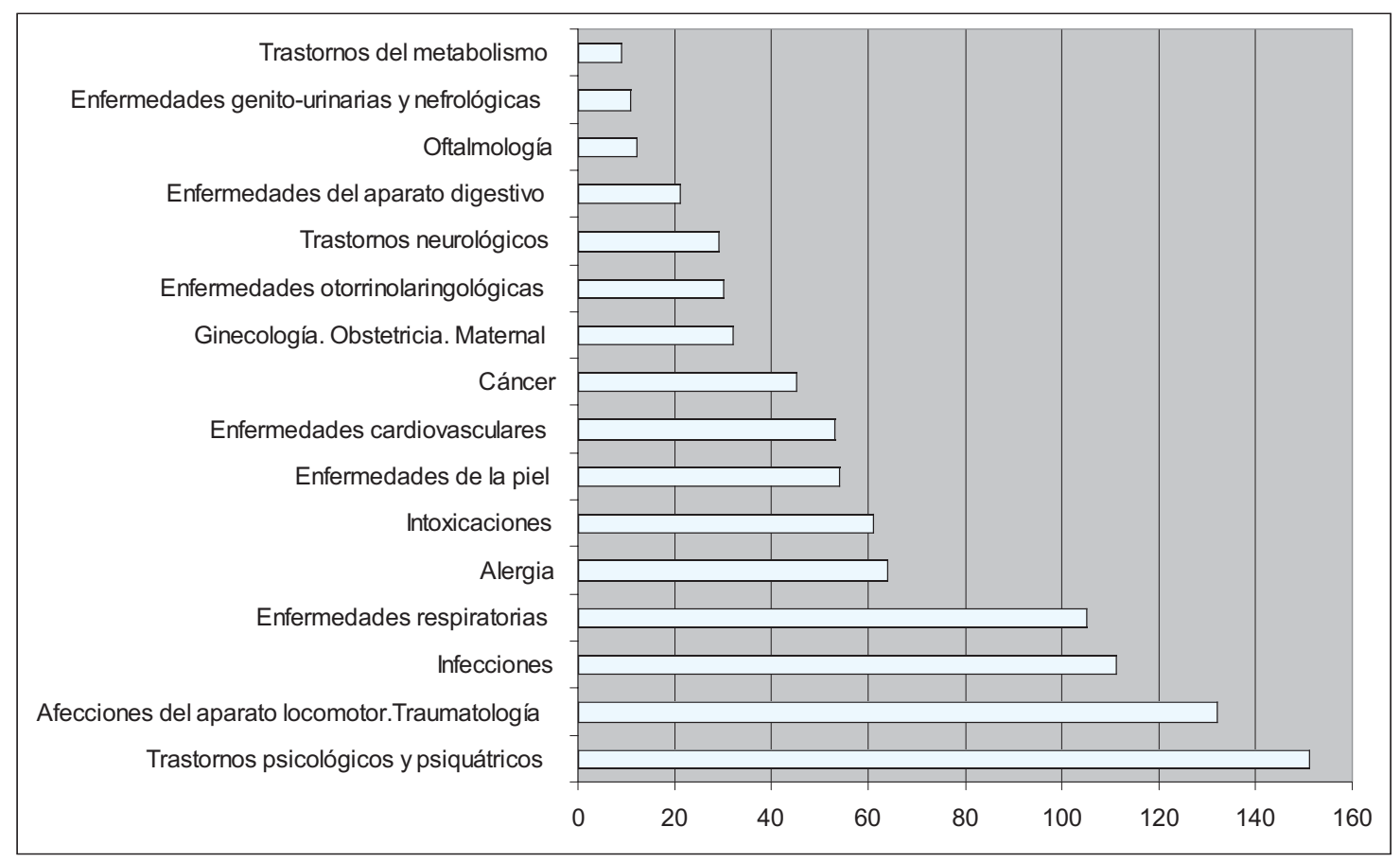

Figura 13. Categoría 2

Categoría 3. Causa o agentes de enfermedad o riesgo laboral

\begin{tabular}{|l|c|}
\hline \multicolumn{1}{|c|}{ GRUPO } & $\mathrm{N}^{\circ}$ DE REGISTROS \\
\hline 31. Agentes químicos & 54 \\
\hline 32. Tóxicos & 107 \\
\hline 33. Agentes físicos & 111 \\
\hline 34. Agentes biológicos & 119 \\
\hline 35. Factores psicológicos & 141 \\
\hline 36. Factores ergonómicos & 84 \\
\hline 38. Hábitos de salud & 53 \\
\hline
\end{tabular}

En cuanto a los factores desencadenantes de los trastornos de la salud laboral, vuelven a destacar los agentes psicológicos, con un total de 241 registros.

\section{Categoría 4. Grupos profesionales}

La categoría laboral más estudiada es la del personal sanitario, con un total de 422 registros, es decir, el $27 \%$ del total de los artículos y el $41 \%$ de los artículos médicos; le sigue muy de lejos la categoría de agricultores y trabajadores agropecuarios y pesqueros, con 41 artículos $(2,6 \%$ del total), y los profesionales de la minería, con un total de 20 artículos $(1,30 \%$ del total $)$.

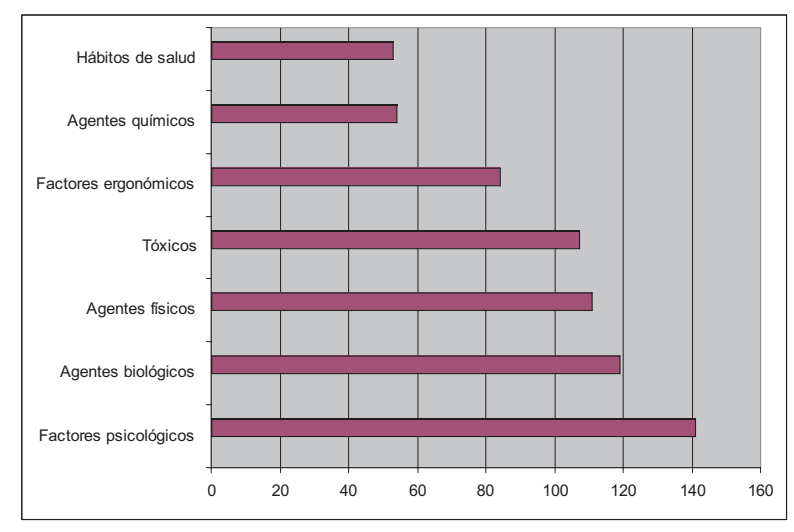

Figura 14. Categoría 3 


\section{CONCLUSIONES}

Respecto a la autoría, hay que considerar el alto índice de autoría colectiva respecto a la individual, lo cual pone de manifiesto la tendencia actual en la ciencia de la existencia de equipos de investigación multidisciplinares.

El índice de autoría múltiple es también elevado, con una media de casi tres autores por artículo.

Es muy significativo el hecho de que prácticamente un $75 \%$ de los autores han publicado un único artículo sobre el tema, lo cual indica un bajo nivel de especialización.

Los autores con un mayor número de aportaciones pertenecen al ámbito hospitalario y de atención primaria, siendo las universidades el segundo grupo más activo.

Analizando las series temporales de producción científica, destaca un aumento de la misma a partir del año 95, coincidiendo posiblemente con una mayor sensibilidad política hacia el tema, con la promulgación de la Ley de Prevención de Riesgos Laborales.

La principal fuente de información de carácter periódico en este campo es la revista Medicina y Seguridad en el Trabajo, una publicación de carácter especializado en esta disciplina; pero llama la atención el hecho de que la segunda y tercera con mayor número de artículos son publicaciones de carácter general, lo cual es otro indicador de la falta de especialización en la materia.
Respecto a las categorías establecidas en el estudio, destaca como fundamental los estudios sobre riesgos, seguido de los aspectos relativos a psicología del trabajo.

En relación con las materias emergentes, se observa la alta producción, en los últimos cuatro años, de los artículos sobre cuestiones relativas a la psicología del trabajo en sentido amplio (estrés profesional, satisfacción laboral, mobbing, etc.)

La categoría profesional más estudiada, con gran diferencia sobre el resto de grupos profesionales, es la del personal sanitario, existiendo así un mismo sujeto y objeto de la investigación.

\section{LÍMITES AL ESTUDIO}

Cualquier trabajo de este tipo, a pesar de contar con una rigurosa metodología, debe de ser tomado con cautela; los datos nunca pueden ser considerados como concluyentes ni definitivos. Se trata de conclusiones relativas, orientativas de una determinada tendencia. Esto responde a diferentes causas, tales como:

- Utilización de una sola fuente de información.

- Deficiencias en la fuente de datos primaria tomada como base del estudio: desigual calidad de los datos, falta de homogeneización, falta de normalización, retraso en la recogida de información.

- Ausencia de normalización en la forma de autores y entidades. Sería recomendable que los investigadores utilizaran siempre una forma unívoca de identificación, según recomienda la FECYT, Fundación Española de Ciencia y Tecnología. 


\section{BIBLIOGRAFÍA}

1. Ley de Prevención de Riesgos Laborales. URL: http://www.boe.es/g/es/bases_datos/doc.php?coleccion=i berlex\&id=1995/24292 [Consultado: 12 de mayo de 2008].

2. Estrategia española de Seguridad y Salud en el Trabajo (2007-2012). URL: http://www.isciii.es/htdocs/centros/medicinadeltrabajo/ultima_hora/EESST.pdf [Consultado: 12 de mayo de 2008].

3. Alba N, Abad F, García C. Frecuencia y características de los artículos españoles sobre enfermedades y accidentes de trabajo a partir de 1a base de datos IME (1971-1996). Archivos de Prevención 1999; 2(1): 4-10.

4. Bases de datos Bibliográficas del CSIC URL: http://bddoc.csic.es:8085/ [Consultado 30 abril de 2008].

5. Medicina y seguridad en el trabajo. URL: http://scielo.isciii.es/scielo.php/script_sci_serial/lng_es/pi d_0465-546X/nrm_iso [Consultado: 12 de marzo de 2008].

6. Medicina clínica. URL: http://db.doyma.es/cgibin/wdbcgi.exe/doyma/mrevista.salta_a_ultimo?pident $=2$ [Consultado: 12 de marzo de 2008].

7. Atención primaria. URL: http://db.doyma.es/cgibin/wdbcgi.exe/doyma/mrevista.salta_a_ultimo?pident $=2$ 7 [Consultado: 12 de marzo de 2008].

8. Archivos de prevención. URL: http://db.doyma.es/ cgi-bin/wdbcgi.exe/doyma/mrevista.salta_a_ultimo? pident $=178$ [Consultado: 12 de marzo de 2008].

9. Revista española de salud pública. URL: http://www.msc.es/resp [Consultado: 12 de marzo de 2008].

10. Gaceta sanitaria. URL: http://www.doyma.es/gs/ [Consultado: 12 de marzo de 2008].

11. Tesauro ILO-CIS. URL: http://www.ilo.org/dyn/ cisdoc/cisthes_search.ilostart?p_lang $=\mathrm{S}$ [Consultado: 12 de mayo de 2008].

12. Clasificación Internacional Uniforme de Ocupaciones. URL: http://www.ilo.org/public/spanish/bureau/ stat/class/isco.htm [Consultado: 12 de mayo de 2008].

13. Recomendaciones para la correcta identificación de las publicaciones científicas. URL: http://www.accesowok.fecyt.es/recomendaciones_publicaciones.html [Consultado el 10 de mayo de 2008].
14. Alonso Arévalo J, Ríos Hilario AB. Características de la investigación en Biblioteconomía y Documentación a partir del análisis de la información recogida en la base de Datos Dathatéke. E-LIS: E-Prints in Library and Information Science 2004. URL: http://eprints.rclis.org/archive/00002522/01/Investiga10.pdf [Consultado el 12 de mayo de 2008].

15. Ardila CS, Ronda E. Las fuentes de información bibliográfica en salud laboral. Archivos de Prevención 2000; 3(1):5-11.

16. Benavides, FG. La salud laboral en España a examen: Informe del Observatorio de Salud Laboral 2006. Archivos de Prevención de Riesgos Laborales. 2007; 10(3):121-122.

17. Carrasco Mallén, M. La investigación: un elemento necesario en las estrategias de seguridad y salud en el trabajo. Medicina y Seguridad en el Trabajo. 2003; L(193):1-3.

18. García Gómez M., Gil López, E. Los sistemas de información y la vigilancia laboral. Revista Española de Salud Pública. 1996; 70(4):393-406.

19. Grupo Scimago. Ranking de instituciones más productivas del sector sanitario español. El Profesional De La Información 2005; 14(5). URL: http://elprofesionaldelainformacion.metapress.com/(j50ltv55nlwsbvu11bndb $\mathrm{gmb} /$ app/home/journal.asp? referrer=parent\&backto=ho memainpublications, 1,1 .

20. Frías Osuna A, Pulido Soto A, Pino Casado RD, Palomino Moral PÁ. Características de la investigación de la calidad en enfermería comunitaria. Enfermería Comunitaria 2006; 2(2):114-24.

21. Jiménez-Contreras E, Faba C, Moya FD. El destino de las revistas científicas nacionales. El caso español a Través de una muestra (1950-90). Revista Española De Documentación Científica 2001; 24(2). URL: http://www.cindoc.csic.es/redc/242/2-01 esp.html.

22. Licea de Arenas J, Arenas M, Cabello M, Carmona $\mathrm{V}$, Romero RD. Investigación mexicana significativa en ciencias de la salud 1999-2004. Un análisis bibliométrico. Anales De Documentación 2006; 9. URL: http://www.um.es/fccd/anales/ad09/ad0908.pdf [Consultado el 12 de mayo de 2008].

23. Llana Martín Adl, Barredo Sobrino MP. Estudio bibliométrico de la producción científica de los departamentos básicos de la Facultad de Medicina de la Universidad Autónoma de Madrid (1990-2000). Universidad 
Autónoma De Madrid 2003. URL: http://biblioteca. uam.es/documentos/cc10.pdf [Consultado el 12 de mayo de 2008]

24. Lorenzo-Cáceres Ascanio Ad, Otero Puime Á. Publicaciones sobre evaluación de la atención primaria en España tras veinte años de reforma (1984-2004): análisis temático y bibliométrico. Revista Española de Salud Pública 2007; 81(2):131-45. URL: http://www.msc.es/ biblioPublic/publicaciones/recursos_propios/resp/revista_cdrom/vol81/vol81_2/RS812C_131.pdf
25. Mapa bibliométrico de España 1996-2000: biomedicina y ciencias de la salud. URL: http:// 84.88.71.251/mapabiomedico2004/Index.htm [Consultado el 12 de mayo de 2008]

26. Torres-Salinas D. Diseño de un sistema de información y evaluación científica: análisis ciencimétrico de la actividad investigadora de la Universidad de Navarra en el área de ciencias de la salud 1999-2005. URL: http://eprints.rclis.org/archive/00011717/ [Consultado el 10 de mayo de 2008]. 